\title{
Superfluorescence from Magnetically Formed Quantum Dots: the Excitation Pulse-Width Dependence
}

\author{
Young-Dahl Jho* \\ Department of Information and Communications, Gwangju Institute of Science and Technology \\ (GIST), Gwangju 500-712, Korea \\ Jinho Lee, Gary D. Sanders, Christopher J. Stanton, and David H. Reitze \\ Department of Physics, University of Florida, Gainesville, FL 32611, USA \\ Junichiro Kono \\ Department of Electrical and Computer Engineering, Rice University, Houston, TX 7r005, USA \\ Alexey A. Belyanin \\ Department of Physics, Texas A\&M University, College Station, TX 77843, USA
}

(Received February 29, 2008 : revised March 18, 2008)

\begin{abstract}
We investigated the laser pulse-width dependence of dense plasmas confined within the magnetic length of $\mathrm{In}_{0.2} \mathrm{Ga}_{0.8} \mathrm{As} / \mathrm{GaAs}$ multiple quantum wells under high magnetic fields up to $31 \mathrm{~T}$. To fully fill the Landau levels of effectively zero-dimensional system, we used intense femtosecond (fs) laser pulses to create carrier densities near $10^{13} / \mathrm{cm}^{2}$. The observed photoluminescence showed a characteristic of superfluorescence, above critical magnetic field when being excited by pulses shorter than coherence buildup time.
\end{abstract}

OCIS codes : $300.6280,320.7120$

\section{INTRODUCTION}

The spontaneous appearance of macroscopic coherence such as Bose-Einstein condensation and superconductivity is among the most dramatic cooperative phenomena in condensed matter physics, and the search for ordered exciton states is the subject of intense current research [1]. In quantum electrodynamics, under specific physical conditions there exists a related selforganization process of the same fundamental importance, called superfluorescence (SF) $[2,3]$. In this process, a system of $N$ inverted atoms is incoherently prepared, but macroscopic coherence builds up self-consistently starting from vacuum fluctuations at a sufficiently high density $N$, after a certain delay time called coherence buildup time $t_{d}$ [3]. The resultant macroscopic dipole decays superradiantly, producing a burst of coherent radiation. The exotic SF pulses have been observed in atomic gases [4,5] and rarefied impurities in crystals $[6,7]$ but only recently observed in semiconductor systems $[8,9]$ because of ultrafast decoherence. As a new route to overcoming this obstacle, we use a magnetic field in order to confine charged carriers within, the so-called, magnetic length $\left(\sim 25 \mathrm{~nm} / B^{1 / 2}\right.$, with magnetic field $B$ in the unit of Tesla) in quantum wells (QWs). As such, we can simulate ideal nano-scale quantum optics in atomic-like environment without disturbance from alloy or size fluctuations. The intense femtosecond (fs) laser pulses create and probe quantum coherence between electrons and holes, combined with a strong perpendicular magnetic field to increase the density of states through quantum-dot-like Landau quantization. The spontaneous formation of a coherent quantum ensemble is fundamentally different from laser-driven coherence. Typically, such transitions occur at a critical temperature and/or density at which the particle interactions energetically favor a single $N$-body wave function.

We previously observed the emission of SF bursts from inter-Landau level (LL) recombination as evidenced from the resulting spectral, spatial, and statistical characteristics of the luminescence [8]. Our investigations revealed a clear transition from a spontaneous band-edge emis- 
sion regime at low carrier densities and magnetic fields to a randomly directed but highly collimated beam in $\mathrm{SF}$ regime at highest densities and magnetic fields. The effects of temperature on the threshold magnetic field for SF was understood based on a phonon model which modifies the coherence time $T_{2}$ through acoustic and optical phonon scattering rates [9]. Here we extend these measurements to investigate how the SF emissions depend on the excitation pulse-width.

\section{EXPERIMENTAL RESULTS AND DISCUSSION}

The experimental setup to perform luminescence measurements simultaneously under intense fs laser excitation and high magnetic fields was newly developed at the National High Magnetic Field Laboratory in Tallahassee, FL, USA. As shown in Fig. 1 (a), we used a $130 \mathrm{fs}, 800 \mathrm{~nm}$ Ti:sapphire chirped pulse amplifier system $(\mathrm{CPA})$, and measured photoluminescence $(\mathrm{PL})$ as a function of magnetic field $B$. The magnetic field was perpendicular to quantum well plane and the emission was collected using optical fibers from the opposite face (center collection geometry) and a cleaved edge (edge collection geometry) of the sample and analyzed with a pair of spectrometer and charge-coupled device detectors. Samples used in this work were grown by molecular beam epitaxy on GaAs substrates, and consisted of a GaAs buffer layer followed by 15 layers of $8 \mathrm{~nm}$ undoped $\mathrm{In}_{0.2} \mathrm{Ga}_{0.8}$ As QW separated by undoped $15 \mathrm{~nm}$ GaAs barriers and a $10 \mathrm{~nm}$ GaAs cap layer. The sample temperature and laser fluence were fixed at $10 \mathrm{~K}$ and at around $0.1 \mathrm{~mJ} / \mathrm{cm}^{2}$, respectively. From our previous report [8], luminescence scaling indicated saturation of $\mathrm{SF}$ emission strength above $0.1 \mathrm{~mJ} / \mathrm{cm}^{2}$. To examine the emission characteristics in regard to ultrafast formation and decay of macroscopic coherence, we stretched the pulse width to $30 \mathrm{ps}$ by using a pulse stretcher composed by a grating pair, a lens pair, and a mirror as cartooned in Fig. 1 (b).

Figure 2 displays experimental (dotted lines) and theoretical (solid lines) spectra of (a) absorption coefficient measured by using low power tungsten lamp (N $<10^{10} / \mathrm{cm}^{2}$ ) and (b) PL pumped by CPA with $130 \mathrm{fs}$ pulse width, for different total carrier densities. The main interband transitions between electronic and heavyhole LLs are denoted by 0-0, 1-1, and 2-2 in Fig. 2. Other various minor peaks were assigned to mixed states between dark and bright quantum levels [10]. The detailed theoretical approaches to obtain the spectra were previously reported [10]. We note Fig. 2 (a) and 2 (b) were measured at different magnetic fields of 25 $\mathrm{T}$ and $30 \mathrm{~T}$ while the energy separation between $0-0$ and 1-1 was even smaller in Fig. 2 (b) at $30 \mathrm{~T}$ which are associated with effective mass renormalization caused by high carrier densities [11]. The peak splitting in theory is due to different spin states under $B$ fields while not resolved in actual measurements.

As the carrier density increases from $10^{11} / \mathrm{cm}^{2}$ (red solid line) to $10^{13} / \mathrm{cm}^{2}$ (black solid line) in Fig. 2 (b), various higher LLs emit the luminescence. By comparing the theoretical traces as a function of carrier densities to experimental curve (gray dotted line), we can estimate the total carrier density to be about $10^{13}$ / $\mathrm{cm}^{2}$. The discrepancy in spectral shape between the experiment and theory at $10^{13} / \mathrm{cm}^{2}$ is because the theoretical curve only includes the spontaneous emission component in clear contrast to amplified sharp features that are prominent at specified LLs in experimental data. In addition, the peak separation at each LL was associated with spin splitting in the theory which wasn't observed in the experiment. We also note that the excited carrier density of $10^{13} / \mathrm{cm}^{2}$ is much higher than the Mott density in GaAs-based quantum well system $\left(10^{11} / \mathrm{cm}^{2}\right)$ [12]. Therefore, this high carrier density of $10^{13} / \mathrm{cm}^{2}$ surely lead to plasmonic state at low magnetic fields. Under high magnetic fields $(25 \sim 30 \mathrm{~T})$ when the

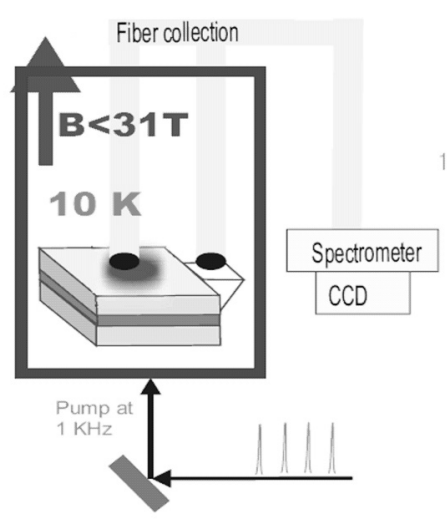

(a)

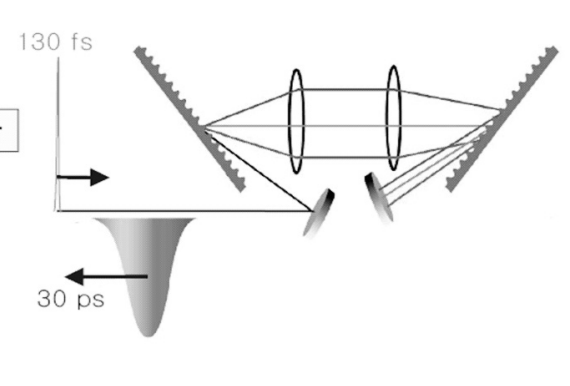

(b)

FIG. 1. (a) Experimental scheme. (b) Pulse stretcher using two wavelength dispersing diffraction gratings. 
magnetic length is reduced by 2 times compared to the zero-field exciton Bohr radius, one could expect plasma rather than excitonic states, comparing the wavefunction size with magnetic length. In this regard, whether the luminescence originates from exciton or plasma needs to be further studied, considering the carrier-carrier interaction under high magnetic fields.

Figure 3 shows emission spectra in edge collection geometry by exciting (a) 130 fs pulse or (b) 30 ps pulse as a function of magnetic fields. The 1-1 LL emission strength compared with 0-0 LL was much stronger in Fig. 3 (a) than in Fig. 3 (b). This indicates a certain amount of slow carrier relaxation from higher LL down to $0-0$ LL exists over the temporal scale of $30 \mathrm{ps}$. We also note the oscillatory behavior at 1-1 and higher LLs. Such oscillatory behavior is related to the enhanced emission strength when the LLs of $2^{\text {nd }}$ quantum confined states are mixed with those of $1^{\text {st }}$ quantum well states and will be fully discussed elsewhere [13].

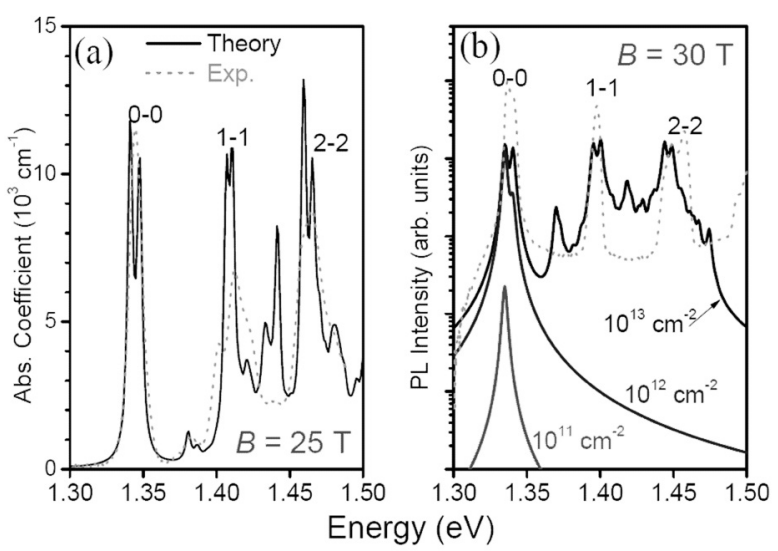

FIG. 2 (a) Absorption coefficient at 30 T: theory (solid line) Vs. experiment (dotted line). (b) Theoretical PL spectra based on spontaneous emission at different carrier densities compared with experiments (dotted line).
In Fig. 4 (a), we traced the peak energy position of sharp Lorentzian feature in 0-0 LL at different pulse width, compared to the case of $\mathrm{CW}$ absorption. The Lorentzian shape is natural for amplified spontaneous emission or $\mathrm{SF}$ processes above $12 \mathrm{~T}$, while the $\mathrm{CW}$ absorption can be well fit by Gaussian. In Fig. 4 (a), the slope of peak position curve was reduced in the case of pulsed excitation due to the effective mass renormalization by sudden high carrier injection [11]. The red-shifted peak position for pulsed excitation also indicates the band gap renormalization $[14,15]$. The effect of band gap renormalization was stronger at 130 fs pulse but the difference between $130 \mathrm{fs}$ and $30 \mathrm{ps}$ was mostly suppressed above $25 \mathrm{~T}$. The 130 fs pulse creates carriers more abruptly than 30 ps pulse and the following carrier-carrier interactions will be enhanced to screen the excitonic Coulomb interactions more effectively. The disappearance of the peak separation between 130

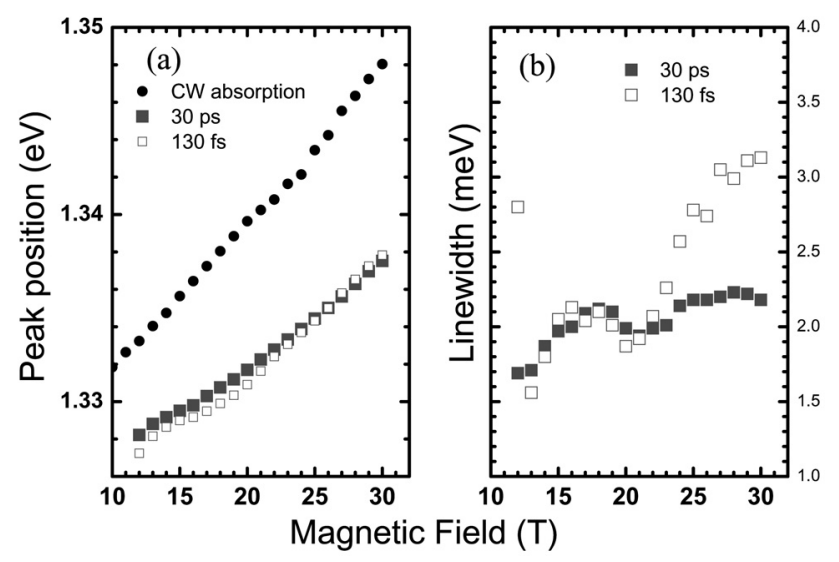

FIG. 4 (a) 0-0 LL peak energy positions for the pulsewidth of 130 fs (open square) and 30 ps (solid square), compared to low power CW absorption. (b) Linewidth of the sharp feature from the 0-0 LL versus B for 130 fs (open square) and for $30 \mathrm{ps}$ (solid square).
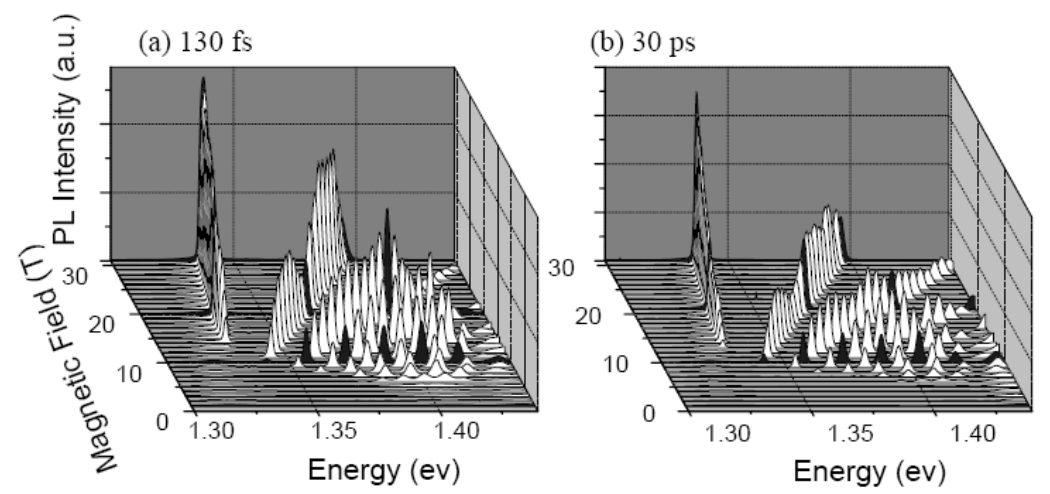

FIG. 3 PL spectra as a function of magnetic fields up to $30 \mathrm{~T}$ for the excitation pulse width of (a) 130 fs and (b) 30 ps. 
fs and 30 ps above $25 \mathrm{~T}$, therefore, could be possibly associated with the combined effects of increased carrier density and reduced screening effects because of more confined wavefunction via $B$ increment. We note that above $25 \mathrm{~T}$ the slope of peak position versus $B$ in Fig. 4 (a) became more similar to CW absorption case, which suggests the reduction of effective mass renormalization in spite of increased carrier density at higher fields.

To investigate the characteristic of the emission in Fig. 4 (b), we obtained linewidth for 130 fs (red) and for $30 \mathrm{ps}$ (blue), following the Lorentzian lineshape analysis. Fig. 4 (b) displays the clear trend of increasing linewidths for $130 \mathrm{fs}$ in contrast to those for 30 ps. 30 ps is much longer than the estimated coherence buildup time for SF in our system $(\sim 10 \mathrm{ps})$ $[3,8]$ and the intra-relaxation time on the order of 500 $\mathrm{fs}$ [16]. Since the increment of linewidth with $B$ was a signature of SF [8], plateau in the linewidth trace for $30 \mathrm{ps}$ implies the saturated amplified spontaneous emission, not reaching SF regime.

\section{CONCLUSION}

In this experiment, we have performed photoluminescence measurements in $\operatorname{In}_{0.2} \mathrm{Ga}_{0.8} \mathrm{As} / \mathrm{GaAs}$ multiple quantum wells in high magnetic fields using intense pulse laser source. The resultant spectra showed the luminescence from an ensemble of high carrier density of $10^{13} / \mathrm{cm}^{2}$. The pulse-width dependence further revealed that the degree of effective mass and bang gap renormalization varies with magnetic field. The linewidth showed increment only for 130 fs while being plateaued for 30 ps. These different characters of linewidth implies that the SF can be effectively generated in our system only for the excitation laser pulse width shorter than the coherence buildup time.

\section{ACKNOWLEDGEMENT}

This work was supported in part by the Korea Research Foundation Grant funded by the Korean Government (MOEHRD) (Grant No. 2007-313-C00218). A portion of this work was performed at the National High Magnetic Field Laboratory, supported by NSF Cooperative Agreement No. DMR-0084173.

*Corresponding author: jho@gist.ac.kr

\section{REFERENCES}

[1] Hui Deng, Gregor Weihs, Charles Santori, Jacqueline Bloch, and Yoshihisa Yamamoto, "Condensation of Semiconductor Microcavity Exciton Polaritons," Science, vol. 298, no. 4, pp. 199-202, 2002; J Keeling, F M
Marchetti, M H Szymańska, and P B Littlewood, "Collective coherence in planar semiconductor microcavities," Semicond. Sci. Technol., vol. 22, no. 5, pp. R1-R26, 2007.

[2] R. Bonifacio and L. A. Luiato, "Cooperative radiation processes in two-level systems: Superfluorescence," Phys. Rev. A, vol. 11, no. 5, pp. 1507-1521, 1975.

[3] A. A. Belyanin, V. V. Kocharovsky, and Vl. V. Kocharovsky, "Collecitve QED processes of electronhole recombination and electron-positron annihilation in a strong magnetic field," Quantum Semiclass. Opt., vol. 9, no. 1, pp. 1-44, 1997; A. A. Belyanin, V. V. Kocharovsky, VI. V. Kocharovsky, and D. S Pestov, "Novel schemes and prospects of superradiant lasing in heterostructures," Laser Phys., vol. 13, no. 2, pp. 161167, 2003.

[4] N. Skribanowitz, I. P. Herman, J. C. MacGillivray, and M. S. Feld, "Observation of Dicke superradiance in optically pumped HF gas," Phys. Rev. Lett., vol. 30, no. 8, pp. 309-312, 1973.

[5] H. M. Gibbs, Q. H. F. Vrehen, and H. M. J. Hikspoors, "Single-pulse superfluorescence in Cesium," Phys. Rev. Lett., vol. 39, no. 9, pp. 547-550, 1977.

[6] R. Florian, L. O. Schwan, and D. Schmid, "Superradiance and high-gain mirrorless laser activity of O2_centers in KCl," Solid State Commun., vol. 42, no. 1, pp. 55-57, 1982.

[7] Michelle S. Malcuit, Jeffery J. Maki, David J. Simkin, and Robert W. Boyd, "Transition from superfluorescence to amplified spontaneous emission,” Phys. Rev. Lett., vol. 59, no. 11, pp. 1189-1192, 1987.

[8] Y. D. Jho, Xiaoming Wang, J. Kono, D. H. Reitze, X. Wei, A. A. Belyanin, V. V. Kocharovsky, Vl. V. Kocharovsky, and G. S. Solomon, "Cooperative Recombination of a Quantized High-Density Electron-Hole Plasma in Semiconductor Quantum Wells," Phys. Rev. Lett., vol. 96, no., pp. 237401-237404, 2006.

[9] Y. D. Jho, X. Wang, J. Kono, D. H. Reitze, X. Wei, A. A. Belyanin, V. V. Kocharovsky, VL. V. Kocharovsky, and G. S. Solomon, "Superfluorescence from Dense Electron-Hole Plasmas under High Magnetic Fields, ' $J$. Mod. Opt., vol. 53, no. 16\&17, pp. 2325-2335, 2006.

[10] Y. D. Jho, F. V. Kyrychenko, J. Kono, X. Wei, S. A. Crooker, G. D. Sanders, D. H. Reitze, C. J. Stanton, and G. S. Solomon, "Role of Coulomb interactions in dark-bright magnetoexciton mixing in strained quantum wells," Phys. Rev. B, vol. 72, no., pp. 45340-45346, 2005.

[11] S. Adams, I. Galbraith, B. N. Murdin, K. W. Mitchell, B. C. Cavenett, C. R. Pidgeon, P. B. Kirby, R. S. Smith, and B. Miller, "Direct measurement of the effective-mass renormalization in n-type modulationdoped $\mathrm{Al}_{0.23} \mathrm{Ga}_{0.77} \mathrm{As} / \mathrm{In}_{0.08} \mathrm{Ga}_{0.92} \mathrm{As} / \mathrm{GaAs}$ quantum wells," Phys. Rev. B, vol. 46, no. 20, pp. 13611-13614, 1992.

[12] A. M. Nemilentsau, G. Ya. Slepyan, and S. A. Maksimenko, "Direct observation of the Mott transition in an optically excited semiconductor quantum well," Phys. Rev. Lett., vol. 94, no., pp. 147403-147407, 2005.

[13] Y. D. Jho, X. Wang, J. Kono, D. H. Reitze, X. Wei, A. A. Belyanin, V. V. Kocharovsky, Vl. V. Kocharovsky, G. S. Solomon, "Time-integrated evidences for superfluorescence from dense electron-hole magneto-plasmas in 
semiconductor quantum wells," in preparation (arxiv: cond-matt/0601483).

[14] L. V. Butov, V. D. Egorov, V. D. Kulakovskii, and T. G. Andersson, "Magnetoluminescence study of manybody effects in a dense electron-hole plasma of strained InxGa1-xAs/GaAs quantum wells," Phys. Rev. B, vol. 46, no. 23, pp. 15156-15162, 1992.

[15] A. Mysyrowicz, D. Hulin, A. Antonetti, A. Migus, W.
T. Masselink, and H. Morkoç, "Dressed excitons in a multiple-quantum-well structure: evidence for an optical Stark effect with femtosecond response time," Phys. Rev. Lett., vol. 56, no. 25, pp. 2748-2751, 1986.

[16] M. C. Tatham, J. F. Ryan, and C. T. Foxon, "Timeresolved Raman measurements of intersubband relaxation in GaAs quantum wells," Phys. Rev. Lett., vol. 63, no. 15, pp. 1637-1640, 1989. 